\title{
First search for Lorentz violation in double beta decay with scintillating calorimeters
}

O. Azzolini, ${ }^{1}$ J. W. Beeman, ${ }^{2}$ F. Bellini,,${ }^{3,4}$ M. Beretta $\odot,{ }^{5,6}$ M. Biassoni, ${ }^{6}$ C. Brofferio,${ }^{5,6}$ C. Bucci, ${ }^{7}$ S. Capelli,,${ }^{5,6}$ L. Cardani, ${ }^{4}$ P. Carniti, ${ }^{5,6}$ N. Casali, ${ }^{4}$ D. Chiesa,${ }^{5,6}$ M. Clemenza, ${ }^{5,6}$ O. Cremonesi, ${ }^{5,6}$ A. Cruciani, ${ }^{4}$ I. Dafinei, ${ }^{4}$ S. Di Domizio ${ }^{8,9}$ F. Ferroni, ${ }^{10,4}$ L. Gironi, ${ }^{5,6}$ A. Giuliani, ${ }^{11}$ P. Gorla, ${ }^{7}$ C. Gotti, ${ }^{5,6}$ G. Keppel, ${ }^{1}$ M. Martinez, ${ }^{3,4, *}$ S. Nagorny, ${ }^{10,7, \dagger}$ M. Nastasi ${ }^{5,6}$ S. Nisi, ${ }^{7}$ C. Nones, ${ }^{12}$ I. Nutini, ${ }^{5,6}$ D. Orlandi, ${ }^{7}$ L. Pagnanini, ${ }^{5,6}$ M. Pallavicini, ${ }^{8,9}$ L. Pattavina, ${ }^{7}$ M. Pavan ${ }^{5,6}$ G. Pessina, ${ }^{6}$ V. Pettinacci, ${ }^{4}$ S. Pirro, ${ }^{7}$ S. Pozzi, ${ }^{5,6}$ E. Previtali, ${ }^{5,6}$ A. Puiu, ${ }^{5,6}$ C. Rusconi ${ }^{7,13}$ K. Schäffner, ${ }^{10,7}$ C. Tomei ${ }^{4}$ M. Vignati, ${ }^{4}$ and A. Zolotarova ${ }^{12, \$}$

${ }^{1}$ INFN Laboratori Nazionali di Legnaro, I-35020 Legnaro (Pd), Italy

${ }^{2}$ Lawrence Berkeley National Laboratory, Berkeley, California 94720, USA

${ }^{3}$ Dipartimento di Fisica, Sapienza Università di Roma, P.le Aldo Moro 2, 00185 Rome, Italy

${ }^{4}$ INFN Sezione di Roma, P.le Aldo Moro 2, 00185 Rome, Italy

${ }^{5}$ Dipartimento di Fisica, Università di Milano - Bicocca, I-20126 Milano, Italy

${ }^{6}$ INFN Sezione di Milano - Bicocca, I-20126 Milano, Italy

${ }^{7}$ INFN Laboratori Nazionali del Gran Sasso, I-67010 Assergi (AQ), Italy

${ }^{8}$ Dipartimento di Fisica, Università di Genova, I-16146 Genova, Italy

${ }^{9}$ INFN Sezione di Genova, I-16146 Genova, Italy

${ }^{10}$ Gran Sasso Science Institute, 67100 L'Aquila, Italy

${ }^{11}$ CSNSM, Université Paris-Sud, CNRS/IN2P3, Université Paris-Saclay, 91405 Orsay, France

${ }^{12}$ IRFU, CEA, Université Paris-Saclay, F-91191 Gif-sur-Yvette, France

${ }^{13}$ Department of Physics and Astronomy, University of South Carolina, Columbia, South Carolina 29208, USA

\section{(Received 12 June 2019; revised manuscript received 5 August 2019; published 6 November 2019)}

\begin{abstract}
We present the search for Lorentz violation in the double beta decay of ${ }^{82} \mathrm{Se}$ with CUPID-0, using an exposure of $9.95 \mathrm{~kg} \times \mathrm{yr}$. We found no evidence for the searched signal and set a limit on the isotropic components of the Lorentz violating coefficient of $\stackrel{\circ}{\text { of }}_{(3)}<4.1 \times 10^{-6} \mathrm{GeV}$ (90\% credible interval). This results is obtained with a Bayesian analysis of the experimental data and fully includes the systematic uncertainties of the model. This is the first limit on $\stackrel{\circ}{\text { of }}^{(3)}$ obtained with a scintillating bolometer, showing the potentiality of this technique.
\end{abstract}

DOI: $10.1103 /$ PhysRevD.100.092002

\section{INTRODUCTION}

The development of a coherent theory capable of unifying quantum mechanics and general relativity is a central goal of contemporary particle physics. Different solutions to this problem hypothesize the existence of unconventional physical phenomena at the Planck scale (approximately $10^{19} \mathrm{GeV}$ ), violating the fundamental symmetries of nature. In particular, several models include the breakdown of Lorentz and charge-parity-time reversal $(C P T)$ symmetries for the sake of a consistent quantum

*Present Address: Fundación ARAID and Laboratorio de Física Nuclear y Astropartículas, Universidad de Zaragoza, C/ Pedro Cerbuna 12, 50009 Zaragoza, Spain.

'Present Address: Queen's University, Physics Department, K7L 3N6 Kingston, Ontario, Canada.

"Present Address: CSNSM, Université Paris-Sud, CNRS/ IN2P3, Université Paris-Saclay, 91405 Orsay, France. gravity description [1]. Since this new phenomenology arises at unreachable energies, a direct observation cannot be performed. Nevertheless, such new physics can impact the Standard Model (SM) predictions as an effective theory characterized by Lorentz symmetry violation (LV) [2], producing sizeable effects in low energy processes like double beta decay. The Standard Model extension (SME) [3-5] is the framework where these effective quantum field operators are described, including both LV and $C P T$-odd operators. LV is included with background fields with nonzero vacuum expectation values, resulting in the spontaneous breaking of space-time symmetry [6]. Neutrino physics is an ideal benchmark to test SME prediction, as many operators affect macroscopic phenomena such as neutrino oscillations [7]. In particular, there exists a countershaded operator with no impact on neutrino velocities, that cannot be investigated in neutrino oscillations or time-of-flight measurements. The countershaded operator 
has mass dimension 3 , is renormalizable, and is odd under $C P T$. The strength of its interaction is given by the four independent components of the $\left(a_{\mathrm{of}}^{(3)}\right)^{\alpha}$ coefficient. The absolute value of these components is proportional to the intensity of LV. The three directional components are labeled as $\boldsymbol{a}_{\mathrm{of}}^{(3)}$, while the anisotropic component is labeled as $\stackrel{\circ}{\text { of }}_{\text {(3) }}$. The former can be studied in experiments directly sensitive to the particle directions, while the latter can be studied when directions are not taken into account. The interactions of neutrinos with this operator modify their quadrimomentum from $q^{\alpha}=(\omega, \boldsymbol{q})$ to $\tilde{q}^{\alpha}=(\omega, \boldsymbol{q}+$ $\left.\boldsymbol{a}_{\text {of }}^{(3)}-\stackrel{\circ}{a}_{\text {of }}^{(3)} \hat{\boldsymbol{q}}\right)[6,8]$. In double beta-decay $(2 \nu \beta \beta)$ experiments measuring only the energy of the two emitted electrons, only $\stackrel{\circ}{{ }^{\circ}}$ of remains as a possible source for LV. The standard $2 \nu \beta \beta$ electrons sum spectrum [9-11] is modified in shape, with a sizable modification parametrized by $\stackrel{\circ}{a}$ of . In this work, we present the search for this deformation in CUPID-0, exploiting the excellent resolution and background rejection capability of our detector to put a limit on the value of $\stackrel{\circ}{a_{\text {of }}}$.

\section{CUPID-0 DETECTOR}

CUPID-0 is an experiment designed to search for the neutrinoless double beta decay $(0 \nu \beta \beta)$ of ${ }^{82} \mathrm{Se}$ $\left(Q_{\text {value }}=2997.9 \pm 0.3 \mathrm{keV}\right.$ [12] $)$ with a calorimetric approach, using the technique of scintillating bolometers. The detector, described in detail in Ref. [13], is composed by $26 \mathrm{ZnSe}$ scintillating crystals (24 enriched at $95 \%$ in ${ }^{82} \mathrm{Se}$ and two with natural isotopic abundance) acting as bolometers and interleaved with high purity germanium bolometric light detectors (LDs). The experiment is operating at a base temperature of $10 \mathrm{mK}$ in hall A of Gran Sasso National Laboratory (Italy). The ZnSe crystals and LD are held in position and thermalized through a mechanical copper structure and Teflon supports. The crystals are surrounded by Vikuity ${ }^{\mathrm{TM}}$ reflective foil, to enhance the light collection. The dual heat/light readout allows us to combine the excellent energy resolution of bolometers with the background rejection capabilities of scintillators. Indeed, CUPID-0 reached the lowest background ever measured in a $0 \nu \beta \beta$ bolometric detector, setting the most stringent limits in the search of the ${ }^{82} \mathrm{Se}$ $0 \nu \beta \beta$ to the fundamental and excited states of ${ }^{82} \mathrm{Kr}$ [14-16]. A comprehensive background model has also been developed for CUPID-0 [17], evaluating and localizing all the possible sources of background for the detector. The understanding of the experimental data obtained with this model allowed us to exploit the high number of $2 \nu \beta \beta$ events for detailed studies on this process. CUPID-0 is therefore a suitable candidate with which to perform the study of the $2 \nu \beta \beta$ spectral shape to evaluate the $\stackrel{\circ}{a}$ of parameter.

\section{LORENTZ VIOLATION IN DOUBLE BETA DECAY}

The inclusion of Lorentz violating $2 \nu \beta \beta$ changes the energy spectrum of the two emitted electrons with an additive term parametrized by $\stackrel{\circ}{a_{\mathrm{of}}}$. The differential spectrum can be expressed with

$$
\begin{aligned}
\frac{d \Gamma}{d E}= & C \cdot F\left(Z, t_{1}\right) \sqrt{t_{1}\left(t_{1}+2\right)}\left(t_{1}+1\right) \\
& \cdot F\left(Z, E-t_{1}\right) \sqrt{E-t_{1}\left(E-t_{1}+2\right)}\left(E-t_{1}+1\right) \\
& \left.\cdot\left[(Q-E)^{5}+10 \cdot \stackrel{\circ}{a_{\mathrm{of}}^{(3)}}(Q-E)^{4}\right)\right] \\
= & C \cdot\left(\frac{d I_{2 \nu, \mathrm{SM}}^{\mathrm{Theo}}}{d E}+10 \cdot \stackrel{\circ}{a_{\mathrm{of}}^{(3)}} \frac{d I_{2 \nu, \mathrm{LV}}^{\mathrm{Theo}}}{d E}\right),
\end{aligned}
$$

where $t_{1}$ is the energy of one of the two emitted electrons, $E$ is the sum of the two emitted electrons kinetic energy, $Q$ is the Q-value of the $2 \nu \beta \beta, C$ is the factor taking into account the nuclear matrix element and normalization constants $[8,18], F$ is the Coulomb correction [19], and $\frac{d I_{2 u, S M}^{\mathrm{Theo}}}{d E}$ and $\frac{d I_{2 L . L V}^{T \text { Th }}}{d E}$ are the SM and LV terms of the decay amplitude. The LV is represented as an additive term, characterized by a different spectral shape and the weight of which is given by $\stackrel{\circ}{a_{\text {of }}}$. In Fig. 1, the simulations of the two $2 \nu \beta \beta$ modes for ${ }^{82} \mathrm{Se}$ are reported. These simulations take into account all

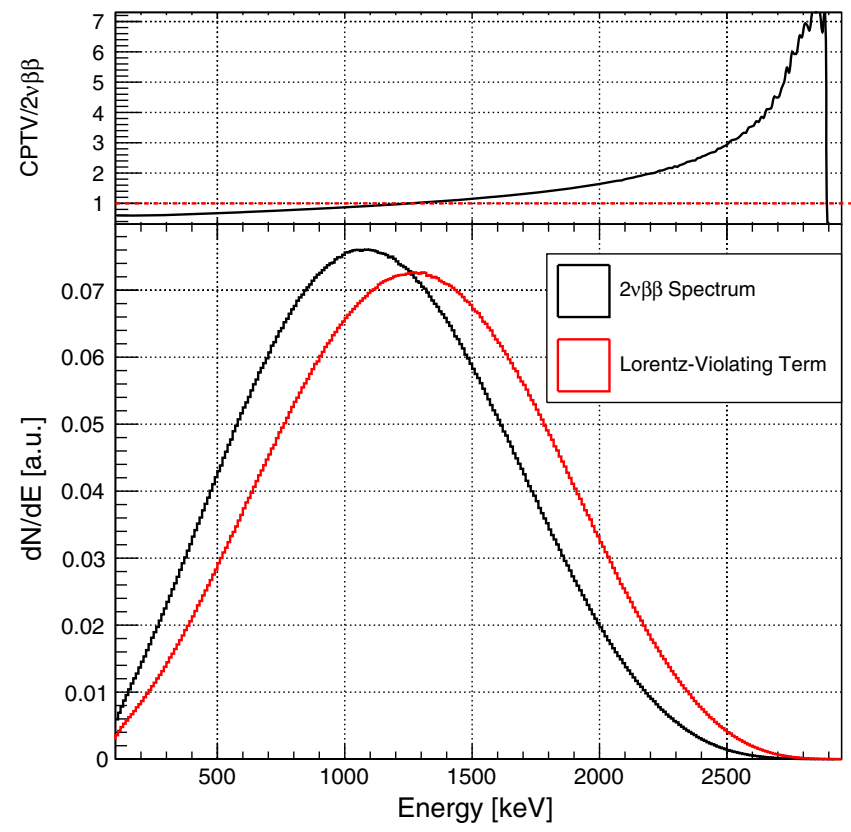

FIG. 1. Comparison between standard (black) and Lorentz violating (red) $2 \nu \beta \beta$ simulated electron spectra for ${ }^{82} \mathrm{Se}$. The simulation is based on exact phase space calculation for $2 \nu \beta \beta[10]$. The emitted electrons are propagated in the detector geometry, and the bremsstrahlung emission is also implemented. The spectra are normalized to the same integral. In the upper panel, the ratio of the two spectra is reported as a function of the energy. 
the inefficiencies in the two electron collection, such as the detector geometry and the bremsstrahlung emission. The $\stackrel{\circ}{a_{\text {of }}}$ parameter can be quantified by comparing the respective integral of the two spectra. The integration of Eq. (1) gives the prediction of the respective weight of the two decay modes in terms of $\stackrel{\circ}{a_{\mathrm{of}}^{(3)}}$ :

$$
\begin{gathered}
C \cdot \int_{0}^{Q} d E \frac{d I_{2 \nu, \mathrm{SM}}^{\mathrm{Theo}}}{d E}=C \cdot I_{2 \nu, \mathrm{SM}}^{\mathrm{Theo}} \\
C \cdot 10 \cdot \stackrel{\circ}{a_{\mathrm{of}}} \cdot \int_{0}^{Q} d E \frac{d I_{2 \nu, \mathrm{LV}}^{\mathrm{Theo}}}{d E}=C \cdot 10 \cdot{\stackrel{\circ}{\mathrm{of}^{(3)}}}^{(3)} I_{2 \nu, \mathrm{LV}}^{\mathrm{Theo}},
\end{gathered}
$$

where $I_{2 \nu, \mathrm{SM}}^{\text {Theo }}$ and $I_{2 \nu, \mathrm{LV}}^{\mathrm{Theo}}$ are the prediction for the standard and Lorentz violating $2 \nu \beta \beta$ modes, respectively. The left sides of Eqs. (2) and (3) can be measured experimentally, and the ratio between the two relations provides a pathway for the evaluation of $\stackrel{\circ}{a}_{\mathrm{of}}^{(3)}$. Defining $\Gamma_{2 \nu, \mathrm{SM}}^{\mathrm{Exp}}$ and $\Gamma_{2 \nu, \mathrm{LV}}^{\mathrm{Exp}}$, respectively, as the standard and Lorentz violating $2 \nu \beta \beta$ measured decay rates, $\stackrel{\circ}{a}$ of can be calculated from

$$
\stackrel{\circ}{a_{\mathrm{of}}^{(3)}}=\frac{1}{10} \frac{I_{2 \nu, \mathrm{SM}}^{\mathrm{Theo}}}{I_{2 \nu, \mathrm{LV}}^{\mathrm{Theo}}} \cdot \frac{\Gamma_{2 \nu, \mathrm{LV}}^{\mathrm{Exp}}}{\Gamma_{2 \nu, \mathrm{SM}}^{\mathrm{Exp}}} .
$$

In this work, the predicted values for $I_{2 \nu, \mathrm{SM}}^{\mathrm{Theo}}$ and $I_{2 \nu, \mathrm{LV}}^{\mathrm{Theo}}$ have been calculated from the integration of Eqs. (2) and (3). The ratio is independent from the matrix element used, since the Lorentz violation in the SME arises from a momentum modification. Therefore, no particular addition had to be taken into account to adapt this evaluation to ${ }^{82} \mathrm{Se}$.

\section{DATA ANALYSIS}

On the measurement side, the evaluation of the standard and Lorentz violating $2 \nu \beta \beta$ was performed with a Bayesian procedure on CUPID-0 experimental data, with a fit of simulated background spectra to the measured data. Data used in this work were collected from June 2017 to December 2018 and correspond to a total $\mathrm{Zn}^{82} \mathrm{Se}$ exposure of $9.95 \mathrm{~kg} \times \mathrm{yr}\left(3.88 \times 10^{25}{ }^{82} \mathrm{Se}\right.$ nuclei $\left.\times \mathrm{yr}\right)$.

Time, amplitude, pulse shape, light output, and other pulse information were extracted from the collected events following the procedure described in Ref. [20]. Selection cuts were applied to the data to exclude nonparticle events, with a total efficiency of $\epsilon=(95.7 \pm 0.5) \%$, constant above $150 \mathrm{keV}$ [17]. The searched signal consists of two electrons detected by a single crystal. Other event categories can be used to constrain the background sources, such as multisite events used to constraint muon-induced showers [17]. Events were then classified according to the nature of the interacting particle $(\alpha$ or $\beta / \gamma)$ and the number of ZnSe crystals that simultaneously triggered the event. The particle identification was exploited only above $2 \mathrm{MeV}$, where the measurement of the different light output allowed us to distinguish $\alpha$ from $\beta / \gamma$ particles [17,20]. Below $2 \mathrm{MeV}$, the poor resolution of light detectors prevented such discrimination. The residual $\alpha$ particles that could not be identified below $2 \mathrm{MeV}$ were added to the $\beta / \gamma$ spectrum, both in measured and simulated data [17]. Counting the number of crystals fired simultaneously in a coincidence window of $20 \mathrm{~ms}$ allowed us to define events in which one, two, or multiple crystals were involved simultaneously. Due to its small statistics, the last category was only used to constrain the background produced by muons. The particle identification and the event multiplicity were used to construct four spectra: single hit spectra of $\alpha$ and $\beta / \gamma$ particles $\left(\mathcal{M}_{1 \alpha}\right.$ and $\left.\mathcal{M}_{1 \beta / \gamma}\right)$, the double hit spectrum $\left(\mathcal{M}_{2}\right)$, and the double hit spectrum where the energy is the sum of the two hit energy $\left(\Sigma_{2}\right)$. The same spectra have also been defined on simulated data, allowing the fit procedure. As previously explained, the large majority of the $2 \nu \beta \beta$ signal events occurs in $\mathcal{M}_{1 \beta / \gamma}$. To describe these spectra, the CUPID-0 background model uses 33 different sources, identified on the basis of experimental data and previous experimental results obtained in the same infrastructure (CUORE-0 experiment [21]). For the analysis described in this paper, the Lorenz violating $2 \nu \beta \beta$ was added to the background model as an additional contribution. The background model was then constructed by a simultaneous fit of the four experimental spectra with a linear combination of the Monte Carlo spectra obtained for the $33+1$ sources. The free parameters of the fit are the activities of each source, parametrized as the coefficients of this linear combination. The measured spectrum of each source is simulated by the means of a Geant4-based Monte Carlo simulation, taking into account the detector geometry and its finite temporal and energetic resolution [17]. The simulation software also addresses the bremsstrahlung emissions of the electrons, which affects the $2 \nu \beta \beta$ spectral shape. A Bayesian approach is chosen to solve this problem $[17,21]$; hence, a prior distribution for each normalization parameter is defined. The priors of the 33 background components are the same as in Ref. [17]. For the Lorentz violating $2 \nu \beta \beta$, a non-negative uniform prior has been chosen, considering this process as an alternative decay channel with respect to the standard $2 \nu \beta \beta$. The joint posterior probability density function (pdf) of all fit parameters has been sampled with JAGS (Just Another Gibbs Sampler) [22], a software based on a Markov chain Monte Carlo algorithm. The joint posterior pdf is subsequently marginalized to obtain the pdf for each normalization parameter. This strategy exploits the relevant experimental signatures of the different background sources to constraint their activities. Both $2 \nu \beta \beta$ modes produce most events in the $\mathcal{M}_{1 \beta / \gamma}$. As a direct consequence, the background sources which are constrained by other spectra, or the normalization of which is anchored to a peak in the experimental spectrum, are not affected by the introduction of Lorentz violating $2 \nu \beta \beta$ in the model. The unaffected sources are: 
(i) both bulk and surface $\alpha$ sources localized in the $\mathrm{ZnSe}$ crystals and in the reflective foil, since their normalization is constrained by the $\mathcal{M}_{1 \alpha}$ spectrum;

(ii) $\gamma$ sources of which the normalization is determined by the intensity of the experimental peaks;

(iii) muons, since they are normalized on the higher multiplicity spectra.

The remaining contribution to the background model, excluding the two $2 \nu \beta \beta$ modes, is represented by ten sources correlated to the searched signature, since they produce a continuum in the $1500-2000 \mathrm{keV}$ energy range. Their effect on the measured coupling constant is subsequently discussed.

To extract the value of $\stackrel{\circ}{a}_{\text {of }}^{(3)}$, the $\Gamma_{2 \nu, \mathrm{LV}}^{\operatorname{Exp}} / \Gamma_{2 \nu, \mathrm{SM}}^{\mathrm{Exp}}$ ratio [see Eqs. (2)-(4)] is computed for each sampling of the joint posterior pdf. In Fig. 2, the experimental $\mathcal{M}_{1 \beta / \gamma}$ spectrum is shown together with the reconstruction. The experimental data are well described by the chosen model, even with the inclusion of the Lorentz violating $2 \nu \beta \beta$. The distribution of fit residuals has a Gaussian shape, with average compatible with zero and $\sigma$ compatible with 1 . In addition, the values of the background sources activities present only small variations with respect to the results reported in Ref. [17]. In particular, the $2 \nu \beta \beta$ activity obtained by this fit is

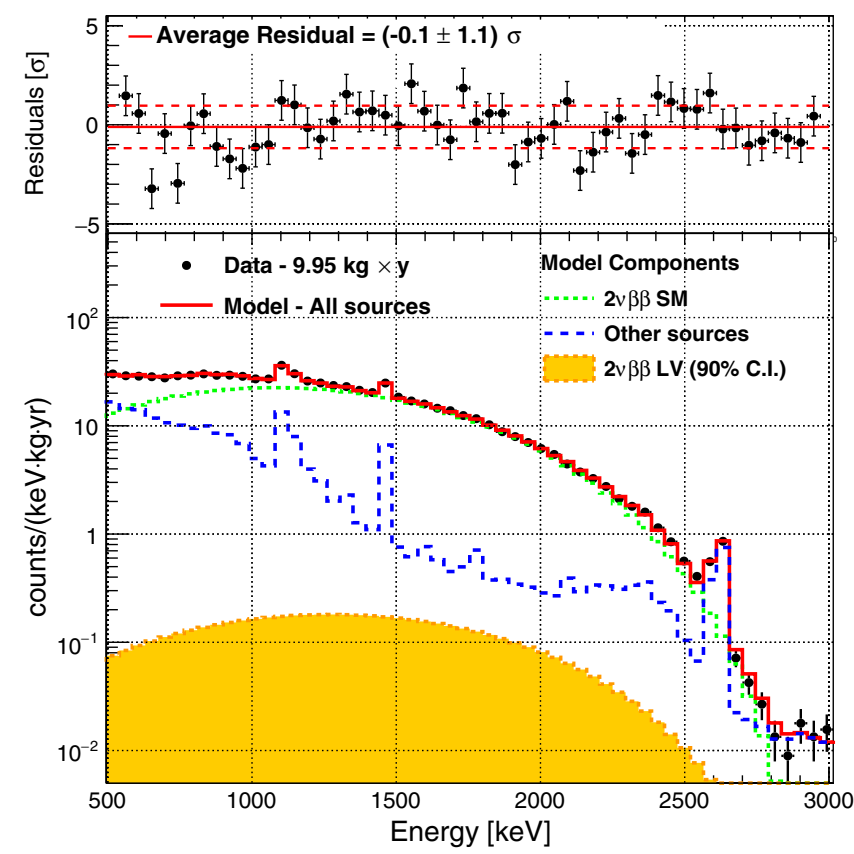

FIG. 2. CUPID-0 $\mathcal{M}_{1 \beta / \gamma}(9.95 \mathrm{~kg} \times \mathrm{yr}$ of $\mathrm{ZnSe}$ exposure) (black dots). The red line shows the reconstruction performed with the fit. The green dashed line is the reconstruction of the standard $2 \nu \beta \beta$, while the orange spectrum is the $90 \%$ CI limit for the Lorentz violating component.The blue dashed line is the sum of all the other 32 background contributions. In the upper panel, the residuals are reported as a function of the energy. The agreement is good over all energies, especially around the peaks, where the model exploits the relevant spectral features to fix its reconstruction.
$(9.8 \pm 0.1) \times 10^{-4} \mathrm{~Bq} / \mathrm{kg}$, while in the background model, it is $(9.96 \pm 0.03) \times 10^{-4} \mathrm{~Bq} / \mathrm{kg}$.

From the posterior distribution for $\Gamma_{\text {Standard }}^{2 \nu} / \Gamma_{\mathrm{LV}}^{2 \nu}(\mathcal{R})$, the distribution for $\stackrel{\circ}{a_{\text {of }}}$ can be calculated, combining the sampled posterior distribution with the theoretical value for the reciprocal weight of the two decay modes [Eqs. (2)-(4)]. The conversion factor from $\mathcal{R}$ to $\stackrel{\circ}{a}_{\text {of }}^{(3)}$ is calculated from Eqs. (1)-(3) as follows:

$$
\frac{I_{2 \nu, \mathrm{SM}}^{\mathrm{Theo}}}{10 \cdot I_{2 \nu, \mathrm{LV}}^{\mathrm{Theo}}}=(213.3 \pm 0.7) \times 10^{-6} \mathrm{GeV},
$$

where the error is due to the uncertainty on the Q-value of ${ }^{82} \mathrm{Se} 2 \nu \beta \beta$. We folded this uncertainty in $\mathcal{R}$, using the Gaussian distribution for $I_{2 \nu, \mathrm{SM}}^{\mathrm{TheO}} /\left(10 \cdot I_{2 \nu, \mathrm{LV}}^{\mathrm{Theo}}\right)$. Since no significant evidence of the Lorentz violating $2 \nu \beta \beta$ could be detected, a 90\% credible interval (CI) limit is determined from the obtained $\stackrel{\circ}{a}$ of distribution.

\section{SYSTEMATICS STUDY}

The posterior pdf is affected by the correlation with the nuisance parameters of the model, i.e., the other normalization coefficients and the influence variables. The correlation with other model parameters is taken into account during the marginalization of the joint posterior distribution. The influence variables instead are arbitrary parameters used in the fit and have to be changed to determine their effect on the analysis result. The bin width used to build the spectra and the lower threshold applied to the data have been considered as influence variables. The following tests have been performed:

(i) Bin: - Bin values of 15, 30, and $50 \mathrm{keV}$ have been used to perform the fit.

(ii) Threshold: - Thresholds of 200, 300, and $500 \mathrm{keV}$ have been used in different fits.

Alongside the influence variables, the hypothesis on the source location in the background model constitutes another possible source of systematic uncertainty. In particular, the positioning of ${ }^{40} \mathrm{~K},{ }^{60} \mathrm{Co}$, and ${ }^{232} \mathrm{Th} /{ }^{238} \mathrm{U}$ has to be taken into account, since these sources produce experimental signatures correlated to the Lorentz violating $2 \nu \beta \beta$. As reported in Ref. [17], the CUPID-0 cryostat model is radially divided by the Roman lead shield in two sections, one internal and one external. ${ }^{40} \mathrm{~K},{ }^{60} \mathrm{Co}$, and ${ }^{232} \mathrm{Th} /{ }^{238} \mathrm{U}$ can be present both inside and outside the Roman lead shield. The ${ }^{232} \mathrm{Th} /{ }^{238} \mathrm{U}$ component can in addition be localized in the Roman lead shield, providing further variability. During the performed tests, each source has been removed from one of the possible locations, resulting in two tests (internal or external) for ${ }^{40} \mathrm{~K}$ and ${ }^{60} \mathrm{Co}$ and three tests for ${ }^{232} \mathrm{Th} /{ }^{238} \mathrm{U}$ (no internal, no external, and no Roman lead). An additional influence on the limit also comes from the presence of an unidentified contamination 
TABLE I. Results of different tests performed to evaluate the systematics effects on the $a_{\text {of }}^{\circ}$ limit. For each row, different values have been tested and combined by adding the relative posterior distribution. The total result is obtained as a limit on the sum of all the family posteriors with equal weight.

\begin{tabular}{|c|c|}
\hline Variable & Result $\left(10^{-6} \mathrm{GeV}\right)$ \\
\hline \multicolumn{2}{|c|}{ Influence variables } \\
\hline Binning & $<3.7$ \\
\hline Threshold & $<3.5$ \\
\hline \multicolumn{2}{|c|}{ Different models } \\
\hline${ }^{40} \mathrm{~K}$ & $<4.0$ \\
\hline${ }^{60} \mathrm{Co}$ & $<3.6$ \\
\hline${ }^{232} \mathrm{Th}$ and ${ }^{238} \mathrm{U}$ & $<4.2$ \\
\hline${ }^{90} \mathrm{Sr}$ & $<5.8$ \\
\hline \multicolumn{2}{|c|}{ Energy scale uncertainty } \\
\hline Calibration correction & $<4.1$ \\
\hline Total & $<4.1$ \\
\hline
\end{tabular}

of pure $\beta$-emitters, in particular, from the $\beta$-decaying isotopes with negligible $\gamma$ emission, long half-life ( $>100 d$ ) and high $Q_{\text {value }}$. The only isotope simultaneously meeting these requirements is the ${ }^{90} \mathrm{Sr}$, a fission product originating the $\beta$-decay sequence ${ }^{90} \mathrm{Sr} \rightarrow{ }^{90} \mathrm{Y} \rightarrow{ }^{90} \mathrm{Zr}$, with $Q_{\text {value }}$ of 546 and $2281 \mathrm{keV}$, respectively. To evaluate the effects of this possible contamination, a test has been performed including ${ }^{90} \mathrm{Sr}$ in the list of sources. As reported in Ref. [15], the energy calibration is affected by bias evaluated over all the interest energies. To control the effects of this bias on the current analysis, an evaluation of the obtained limit is performed using the corrected energy scale. For each test, the model shows a satisfactory agreement with data. The fit residual distribution can always be modeled with a Gaussian with mean value compatible with 0 and $\sigma$ compatible with 1 .

\section{RESULTS}

The results of the different tests have been combined in each category by adding the relative posterior distribution functions for ${ }_{\text {of }}^{\circ}$, according to the law of total probability. A uniform prior for each test has been considered, resulting in an average of the distributions in each test family. The corresponding $90 \%$ CI limits on $\stackrel{\circ}{\text { of }}^{(3)}$ are reported in Table I. To obtain a final limit taking into account all the studied effects, the posteriors for each test family have been added with equal weight. The final limit has the value of $\stackrel{\circ}{a_{\text {of }}}<4.1 \times 10^{-6} \mathrm{GeV}$. The final posterior distribution is shown in Fig. 3, with the evaluated 90\% CI limit.

The obtained result establishes a bound for $\stackrel{\circ}{\text { of }}$ (3) $^{\text {, obtained }}$ for the first time with the shape analysis of the $2 \nu \beta \beta$ spectrum measured with a scintillating bolometer. Even with a limited exposure of $9.95 \mathrm{~kg} \times \mathrm{yr}$, the performances of CUPID-0 scintillating bolometers allowed us to reach a

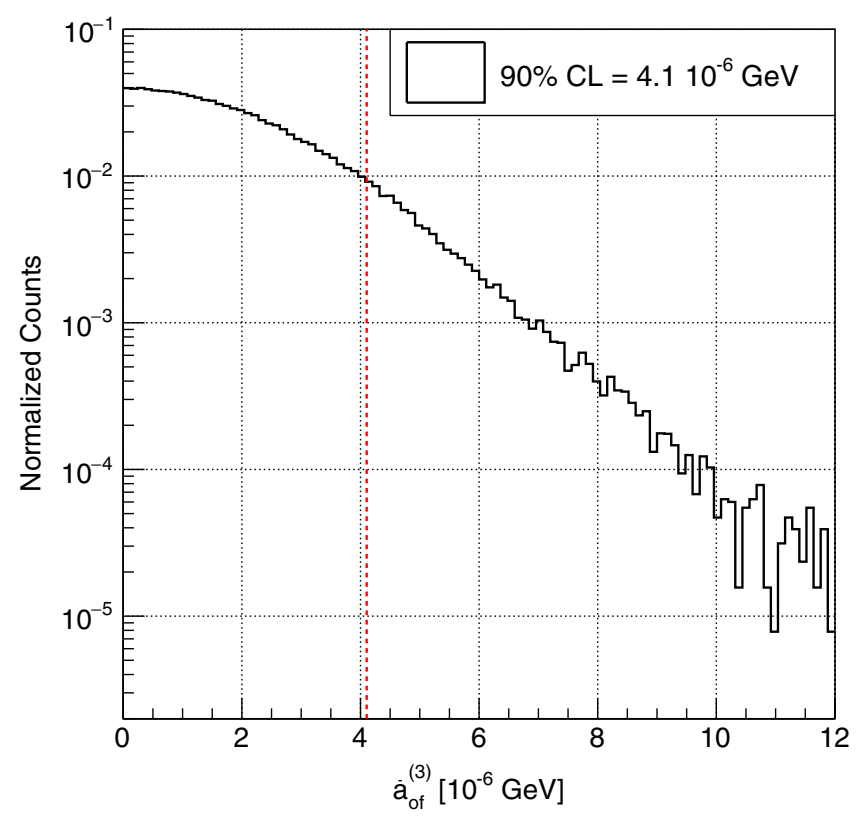

FIG. 3. Total posterior probability distribution for $\stackrel{\circ}{\text { of }}$. The $^{(3)}$. The dashed line represents the $90 \%$ credible interval, corresponding to $\stackrel{\circ}{\text { of }}_{(3)}<4.1 \times 10^{-6} \mathrm{GeV}$.

limit competitive with previously published ones by the EXO-200 Collaboration $\left(\stackrel{\circ}{a}_{\text {of }}^{(3)}<7.6 \times 10^{-6} \mathrm{GeV}\right.$, with an exposure of $100 \mathrm{~kg} \cdot \mathrm{yr}$ ) [18] and NEMO-3 Collaboration $\left(\stackrel{\circ}{a}\right.$ of $<3.5 \times 10^{-7} \mathrm{GeV}$, with $\left.34.4 \mathrm{~kg} \cdot \mathrm{yr}\right)$ [23]. The potentiality of Bayesian analysis applied to bolometric experimental data, showed in Refs. [17,21], is further established.

This result proves that scintillating bolometers can perform spectral shape studies with high sensitivity, even when using a limited statistics. As a consequence, the development of high exposure detectors based on this technique can provide tools to overcome current detectability limits. In addition, this approach can be used to study the Lorentz violation in different $2 \nu \beta \beta$ decaying isotopes. Changing the studied crystals, in fact, allows us to perform the same analysis on different isotopes, with comparable sensitivities [24].

\section{ACKNOWLEDGMENTS}

This work was partially supported by the European Research Council (FP7/2007-2013) under LUCIFER Contract No. 247115. The work of J. K. was supported by the Academy of Finland (Grant No. 314733). We thank M. Iannone for his help in all the stages of the detector assembly, A. Pelosi for constructing the assembly line, M. Guetti for the assistance in the cryogenic operations, R. Gaigher for the mechanics of the calibration system, M. Lindozzi for the cryostat monitoring system, M. Perego for his invaluable help in many tasks, and the mechanical 
workshop of LNGS (E. Tatananni, A. Rotilio, A. Corsi, and B. Romualdi) for the continuous help in the overall setup design. We acknowledge the Dark Side Collaboration for the use of the low-radon clean room. This work makes use of the DIANA data analysis and APOLLO data acquisition software, which was developed by the
CUORICINO, CUORE, LUCIFER and, CUPID-0 Collaborations. This work makes use of the Arby software for Geant4-based Monte Carlo simulations, which was developed in the framework of the Milano Bicocca Research and Development activities and is maintained by O. Cremonesi and S. Pozzi.
[1] G. Amelino-Camelia, Living Rev. Relativity 16, 5 (2013).

[2] J. S. Daz, A. Kostelecký, and R. Lehnert, Phys. Rev. D 88, 071902 (2013).

[3] D. Colladay and V. A. Kostelecký, Phys. Rev. D 55, 6760 (1997).

[4] D. Colladay and V. A. Kostelecký, Phys. Rev. D 58, 116002 (1998).

[5] V. A. Kostelecký, Phys. Rev. D 69, 105009 (2004).

[6] V. A. Kostelecký and J. Tasson, Phys. Rev. Lett. 102, 010402 (2009).

[7] V. A. Kostelecký and N. Russell, Rev. Mod. Phys. 83, 11 (2011).

[8] J. S. Diaz, Phys. Rev. D 89, 036002 (2014).

[9] A. S. Barabash, arXiv:1907.06887.

[10] J. Kotila and F. Iachello, Phys. Rev. C 85, 034316 (2012).

[11] J. Barea, J. Kotila, and F. Iachello, Phys. Rev. C 87, 014315 (2013).

[12] D. L. Lincoln, J. D. Holt, G. Bollen, M. Brodeur, S. Bustabad, J. Engel, S. J. Novario, M. Redshaw, R. Ringle, and S. Schwarz, Phys. Rev. Lett. 110, 012501 (2013).

[13] O. Azzolini et al. (CUPID-0 Collaboration), Eur. Phys. J. C 78, 428 (2018).
[14] O. Azzolini et al. (CUPID-0 Collaboration), Phys. Rev. Lett. 120, 232502 (2018).

[15] O. Azzolini et al. (CUPID-0 Collaboration), Phys. Rev. Lett. 123, 032501 (2019).

[16] O. Azzolini et al. (CUPID-0 Collaboration), Eur. Phys. J. C 78, 888 (2018).

[17] O. Azzolini et al. (CUPID-0 Collaboration), Eur. Phys. J. C 79, 583 (2019).

[18] J. B. Albert et al. (EXO-200 Collaboration), Phys. Rev. D 93, 072001 (2016).

[19] W. C. Haxton and G. J. Stephenson, Prog. Part. Nucl. Phys. 12, 409 (1984).

[20] O. Azzolini et al. (CUPID-0 Collaboration), Eur. Phys. J. C 78, 734 (2018).

[21] C. Alduino et al. (CUORE Collaboration), Eur. Phys. J. C 77, 13 (2017).

[22] M. Plummer, JAGS Version 4.3.0, User Manual, 2012.

[23] R. Arnold et al. (NEMO-3 Collaboration), Eur. Phys. J. C 79, 440 (2019).

[24] I. Nutini, The CUORE experiment: Detector optimization and modelling and $C P T$ conservation limit, Ph. D. thesis, Gran Sasso Science Institute, 2018. 